\title{
Modellierung und Messung von Permafrosttemperaturen im Gipfelgrat der Zugspitze, Deutschland
}

\author{
Jeannette Nötzli, Stephan Gruber, Zürich, Andreas \\ von Poschinger, München
}

\section{Einleitung}

Die Zugspitze im Wettersteingebirge ist mit $2962 \mathrm{~m}$ ü.M. der höchste Gipfel Deutschlands und ein wichtiges Ausflugsziel mit drei Seilbahnen und Bauten sowohl für Sommer- wie auch Wintertouristen (Abb. 1). In der Zugspitze sind die wahrscheinlich bedeutendsten Permafrostvorkommen Deutschlands zu finden. Die wenigen existierenden Studien deuten darauf hin, dass der Fels auf der Nordseite des von Ost nach West verlaufenden Gipfelgrates (Abb. 2) im Untergrund permanent gefroren ist, die Südseite jedoch grösstenteils positive Temperaturen aufweist (GUDE \& BARSCH 2005; Ulrich \& KING 1993). Die Kombination von Permafrost im Grenzbereich seines Vorkommens mit steilen und auf Änderungen schnell reagierenden Felsgraten (NöTZLI \& GRUBER 2009) und bedeutender Infrastruktur macht die Zugspitze zu einem sensitiven Gebiet bezüglich Permafrostdegradation und einer dadurch möglichen Abnahme der Felsstabilität (z.B. HaEberLi et al. 1997). Am 6. Oktober 2001 ereignete sich auf der Nordseite der Vollkarspitze (ca. $4 \mathrm{~km}$ Luftlinie vom Gipfel) auf $2630 \mathrm{~m}$ ü.M. ein Felssturz mit einem Volumen von ca. $30^{\prime} 000 \mathrm{~m}^{3}$, wodurch Teile des Klettersteigs «Jubiläumsgrat» beschädigt wurden (Mustafa 2001) Der Anriss liegt nach einer einfachen Abschätzung im Bereich von warmem Permafrost, das heisst nur wenig unter $0{ }^{\circ} \mathrm{C}$ (NöTzLI et al. 2003).

Um Permafrosttemperaturen im Gipfelbereich der Zugspitze zu untersuchen und langfristig zu beobachten, wurde vom Bayerischen Landesamt für Umwelt (LfU) im Jahr 2006 eine Bohrung geplant. Als Grundlage zur Bestimmung deren Lage wurden in einer Vorstudie die Permafrostverhältnisse mit einem Computermodell simuliert (vgl. Originalbericht von NöTzLI et al. 2006). Die Bohrung wurde im Sommer 2007 realisiert. Damit bietet sich die seltene Möglichkeit, Resultate einer Modellstudie nachträglich mit Messdaten zu überprüfen. In diesem Artikel werden das Modellierverfahren und die Resultate der Vorstudie beschrieben, und es wird ein Überblick über die gemessenen Bohrlochtemperaturen präsentiert. Diese werden mit den Resultaten der Vorstudie sowie einer zusätzlichen erweiterten Simulation verglichen und beurteilt. Die Fragen, die im Zentrum dieses Artikels stehen, sind:
Wie sind die Temperatur- und Permafrostverhältnisse im Gipfelgrat der Zugspitze? Wie gut stimmen die simulierten mit den gemessenen Temperaturbedingungen überein? Welches sind die Vor- und Nachteile sowie Grenzen der angewandten Methode zur Bestimmung einer Bohrstelle?

\section{Hintergrund}

\subsection{Untersuchungsgebiet Zugspitze}

Die Zugspitze ist Teil des Wettersteingebirges in den Bayrischen Alpen und besteht hauptsächlich aus massivem Wettersteinkalk der mittleren alpinen Trias (KöRNER \& UlRICH 1965; UlRICH \& KING 1993). Am Zugspitzgipfel laufen drei lange Felsgrate zusammen: der Wettersteingrat im Südwesten, der Waxensteingrat im Nordosten und der von Ost nach West verlaufende Jubiläumsgrat (Abb. 2). Die Felstopographie fällt auf der Nordseite $60-70^{\circ}$ steil ab, im Süden ist sie flacher mit etwa $35^{\circ}$. Der Kalkstein im Gipfelbereich ist stark verwittert und zerklüftet, und die Hauptrichtung der Kluftsysteme verläuft Richtung NNE und quer zum Grat. Unterhalb des Gipfels auf der Südseite befindet sich das Zugspitzplatt, ein leicht geneigtes Kar mit zwei kleinen Gletschern und Skiliftanlagen.

Die mittlere jährliche Lufttemperatur im Zugspitzgebiet von 1901 bis 2000 beträgt $-4.6^{\circ} \mathrm{C}$ (GUDE \& BARSCH 2005) und beträgt für den modellierten Zeitraum 20002005 (vgl. Abschnitt 3.2) - $4.1^{\circ} \mathrm{C}$ (Datenquelle: Station Zugspitze des Deutschen Wetterdienstes, DWD). Permafrost wurde erstmals beim Bau der Seilbahn im Jahr 1961 entdeckt (UlRICH \& KING 1993). Dabei ist man auf gefrorene Sedimente und bis zu $10 \mathrm{~cm}$ dicke Eiskörper in Klüften gestossen. Beim Bau weiterer Seilbahnanlagen und des Zugtunnels zum Zugspitzplatt in den 80er und frühen 90er Jahren wurde ebenfalls massives Eis gefunden. Den ersten Überblick über die potentielle räumliche Verteilung des Permafrosts im Zugspitzgebiet geben GUdE \& BARSCH (2005) und Mustafa et al. (2003). Die Berechnungen zeigen, dass die Permafrostgrenze wahrscheinlich entlang des Ost-West orientierten Grates verläuft: Die Nordseite des Grates ist Permafrostgebiet, auf der Südseite ist dagegen nur lokal mit Permafrost zu rechnen. Das Zugspitzplatt ist weitgehend frei von Permafrost. Die Berechnungen wurden mit Messungen der basalen Schneetemperatur (BTS) und der Oberflächentemperatur verglichen. 


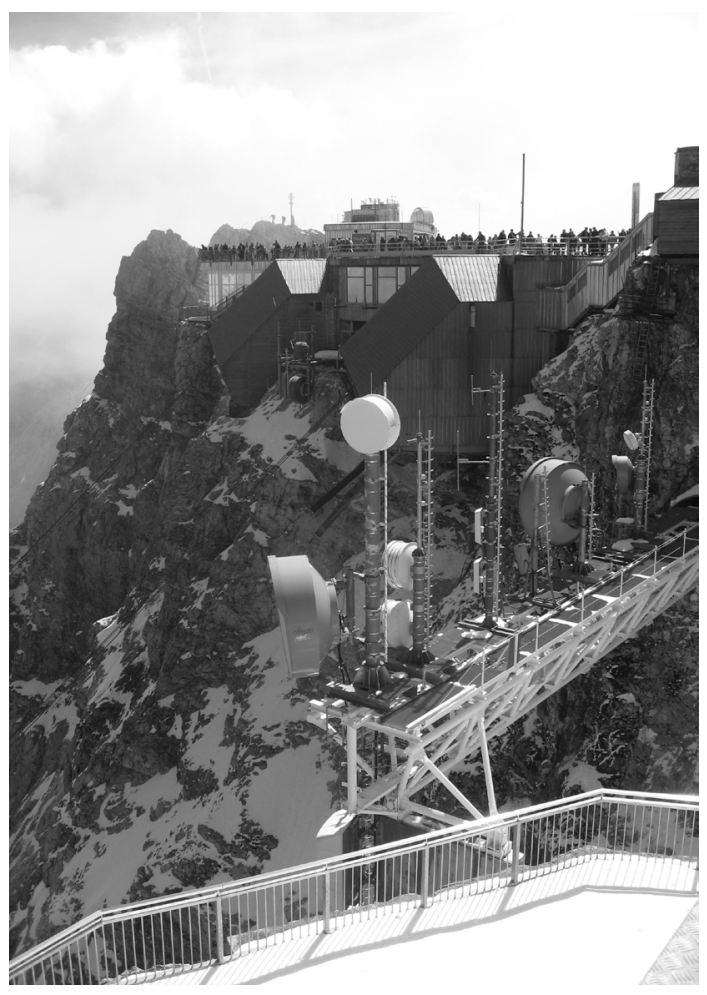

Abb. 1: Bauten auf dem Felsgrat des Zugspitzgipfels Infrastructure on the rock crest of the Zugspitze summit

Constructions sur la crête rocheuse au sommet $d u$ Zugspitze

Foto: J. NöTZLI

\subsection{Felspermafrost}

Hochgebirgsregionen wie die Alpen bestehen zu einem grossen Teil aus steilem Fels, von dem wiederum viel im Permafrost liegt (Gruber \& Haeberli 2007). Die Gebirgspermafrostforschung hat sich im letzten Jahrzehnt verstärkt mit steilen Felswänden beschäftigt. Einerseits ist dies eine Folge zunehmender Beobachtungen von Felsstürzen aus Permafrostgebieten (FISCHER 2009; NöTZLI et al. 2003) und damit einhergehendem steigendem Interesse von Öffentlichkeit und Wissenschaft. Andererseits wurden in den letzen Jahren Messmethoden und Modelle entwickelt (Gruber et al. 2003, 2004; Hasler et al. 2008; WegMANN et al. 1998), die Permafrostforschung im steilen Fels ermöglichen. In den Alpen existiert beträchtliche Infrastruktur, die den Zugang zu diesen Gebieten erleichtert, gleichzeitig aber auch ein erhebliches Schadenpotential darstellt.
Die Permafrostverhältnisse in steilen Felsgebieten werden weitgehend durch die Oberflächentemperaturen, die Untergrundeigenschaften und die Topographie bestimmt. Die Felsoberflächentemperaturen resultieren aus der Oberflächenenergiebilanz. Den grössten Einfluss haben dabei die Sonneneinstrahlung und die Lufttemperatur. Da steile Felswände kaum Schuttbedeckung und im Winter keine wesentliche Schneedecke haben, sind sie an der Oberfläche direkt mit der Atmosphäre gekoppelt. Ihre Reaktion auf veränderte Temperaturbedingungen - zum Beispiel auf eine Hitzewelle wie sie 2003 in Europa herrschte - erfolgt sehr schnell (GRUBER et al. 2004).

Die Hochgebirgstopographie führt zu einer extrem hohen vertikalen und lateralen Variabilität von lokalem Klima, Schneebedeckung, Oberflächen- und Untergrundcharakteristika und damit der Oberflächen- und Untergrundtemperaturen. Gebirgspermaforst ist deshalb sehr inhomogen verteilt, besitzt eine stark dreidimensionale Struktur im Untergrund, und Isothermen sind oft steil, wobei sich Temperaturen nicht in erster Linie mit der Distanz zur Oberfläche ändern, sondern mit der Position zwischen verschieden warmen Seiten eines Gipfels oder Grates (NöTZLI et al. 2007). Die steile Topographie hat einen beschleunigenden Einfluss auf die Geschwindigkeit, mit welcher eine Temperaturänderung an der Oberfläche in den Untergrund eindringt, indem sich Gipfel und Grate von mehreren Seiten erwärmen (NöTZLI \& GRUBER 2009). Zusammen mit der direkten Kopplung von Fels und Atmosphäre und dem im Vergleich zu Schutthalden sehr geringen Eisgehalt führt dies zu besonders schnellen Reaktionen auf Klimaänderungen.

Der Zusammenhang von Permafrostdegradation und Felsstabilität ist noch nicht im Detail verstanden und wird intensiv erforscht, wird aber durch Beobachtungen von massivem Eis in Felssturzanrisszonen kurz nach dem Ereignis bestätigt (FISCHER et al. 2006; KELLER 2003; Ravanel 2006; Sosio et al. 2008). Mögliche Prozesse, welche die stabilitätsrelevanten Bedingungen in eisgefüllten Klüften beeinflussen können, sind das Schmelzen von verbindendem Eis in Klüften (Eiszement) beziehungsweise abnehmende Festigkeit von eisgefüllten Klüften mit steigender Eistemperatur, die Erweiterung von Klüften durch Eissegregation, Volumenexpansion durch Gefrieren von eindringendem Wasser oder der Aufbau von Wasserdruck durch eindringendes Schmelzwasser (GRUBER \& HAEBERLI 2007).

\section{Simulierte Untergrundtemperaturen der Vorstudie}

\subsection{Modellieransatz}

Für die 3D-Simulation der Permafrostverhältnisse im Gipfelgrat wurde der von NöTzLI (2008) entwickelte 


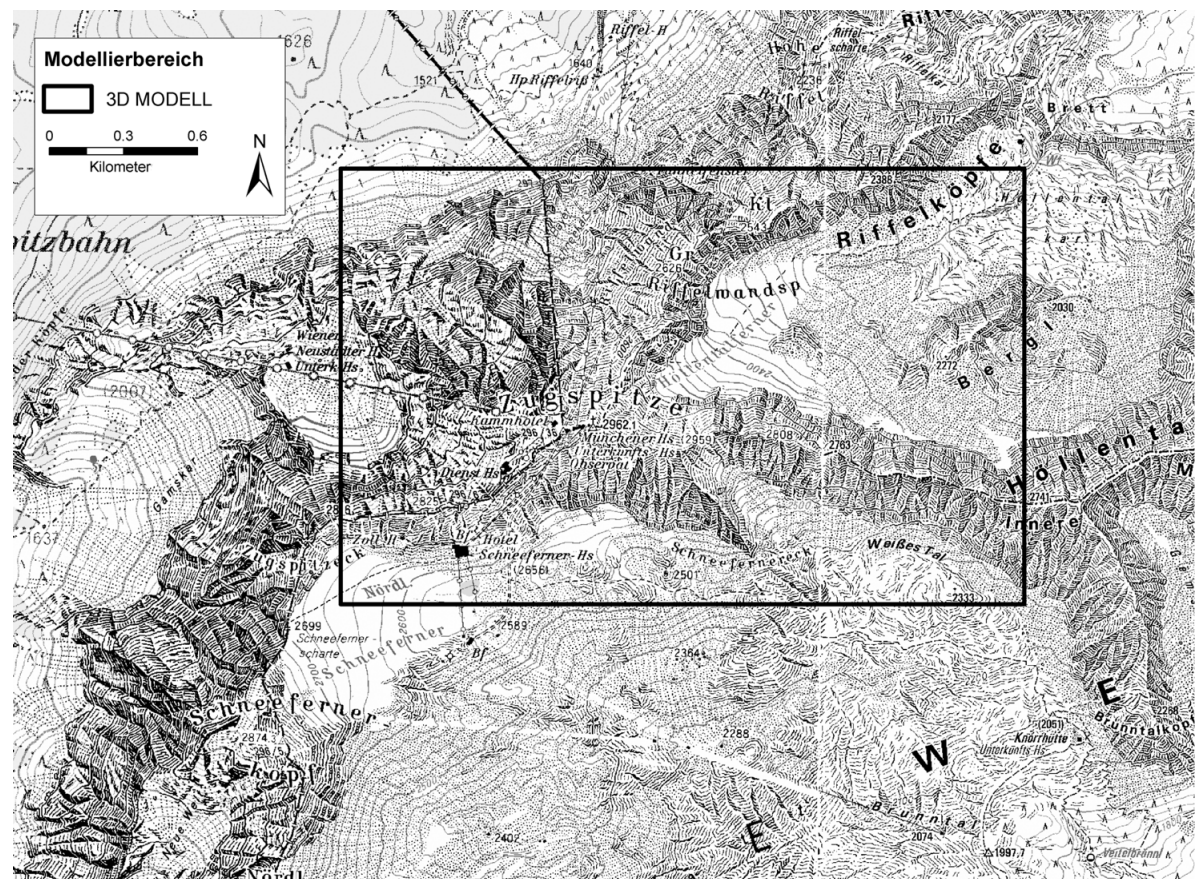

Abb. 2: Übersicht über das Gebiet der Zugspitze. Schwarz eingezeichnet ist der Bereich für die 3D-Modellierung. Overview of the Zugspitze region. The black box marks the area of the 3D-temperature modeling.

Vue d'ensemble de la région du Zugspitze. Le cadre noir indique la région couverte par la modélisation $3 D$ des températures.

Quelle: Topographische Karte 1:25’000 (TOPO25), zur Verfügung gestellt vom Bayerischen Landesamt für Umwelt

und getestete Modellieransatz verwendet. Zuerst wurden mit einem Energiebilanzmodell (GRUBER 2005) über einem digitalen Geländemodell (DGM) die Mitteltemperaturen der Oberfläche berechnet. Als Grundlage dienen Zeitreihen von gemessenen Meteodaten, die mittels speziell für steile Topographie entwickelter Methoden ins Gelände extrapoliert werden. Für jeden Punkt und Zeitschritt werden die Energieflüsse und Oberflächentemperaturen berechnet. Das Energiebilanzmodell wurde validiert für oberflächennahe Felstemperaturen in den Alpen, eine allfällige dünne Schneebedeckung wird dabei nicht berücksichtigt. Stündliche Zeitschritte erlauben es, auch durch den Tagesgang hervorgerufene Effekte zu simulieren. Dies betrifft hauptsächlich unterschiedliche Temperaturen in Ost- und Westexpositionen aufgrund konvektiver Bewölkung.

Die Untergrundtemperaturen wurden in der Vorstudie für eine thermische Gleichgewichtssituation, d.h. ohne Berücksichtigung von zeitabhängigen Effekten
(Veränderungen der Oberflächentemperatur), und unter Berücksichtigung von rein konduktivem Wärmetransport bestimmt. Der 3D-Wärmetransfer im Untergrund wurde mittels der Technik der Finiten Elemente im Programmpaket COMSOL Multiphysics (COMSOL AB, Stockholm) simuliert. Dieses erlaubt ein der Hochgebirgstopographie angepasstes Netz von Finiten Elementen. Das Netz weist eine zunehmende Verfeinerung der Elemente in Richtung der Oberfläche auf. Unterhalb der erstellten Geometrie wurde ein grob diskretisierter $1000 \mathrm{~m}$ hoher Quader angefügt, ohne seitlichen Wärmefluss und mit konstanter unteren Randbedingung von $0.08 \mathrm{Wm}^{-2}$ (MEDICI \& RYBACH 1995).

Die berechneten Oberflächen- und Untergrundtemperaturen sind als Annäherung an reale Temperaturen $\mathrm{zu}$ verstehen und dienen der Abschätzung der thermischen Verhältnisse und deren Verteilung. Da kein Schnee berücksichtig wurde, macht die Interpretation nur für steile Felspartien Sinn, und der flache, schutt- 
bedeckte Teil der Südseite ist aufgrund der teilweise mächtigen Schneedecke wahrscheinlich zu kalt modelliert. Die Hauptunsicherheiten der simulierten Temperaturen sind: (i) Die berücksichtigte Periode 20002005 (vgl. unten) umfasst im Vergleich mit den letzten Dekaden überdurchschnittlich warme Jahre, (ii) die Zunahme der Oberflächentemperaturen seit der Kleinen Eiszeit ist noch nicht in grössere Tiefe vorgedrungen, und transiente Effekte sind wahrscheinlich, und (iii) eine leichte Kühlung ist durch eine dünne Schneedecke möglich, die auch in steilem Fels liegen bleiben kann. Aus diesen Gründen weisen die modellierten Temperaturen wahrscheinlich zu hohe Werte auf. Um die Unsicherheiten zu verdeutlichen und einen möglichen Fehler- oder Schwankungsbereich der berechneten Verhältnisse aufzuzeigen, wird für die Vorstudie zusätzlich eine Simulation der Untergrundtemperaturen für $-2{ }^{\circ} \mathrm{C}(\mathrm{m} 2)$, respektive $+1{ }^{\circ} \mathrm{C}(\mathrm{p} 1)$ abweichende Oberflächentemperaturen gemacht (vgl. NöTZLI et al. 2006). Es wird vermutet, dass die realen Temperaturen am ehesten dem kälteren Szenario entsprechen.

Für den Vergleich der Modellresultate mit den Bohrlochdaten wurde zusätzlich eine transiente Simulation durchgeführt, die Änderungen der Oberflächentemperaturen der letzten 150 Jahre mit einbezieht. Dazu wurden die Änderungen der Lufttemperatur seit der Kleinen Eiszeit rekonstruiert und in der Modellierung als Änderungen der oberen Randbedingungen vorgegeben (vgl. dazu NötZli \& Gruber 2009). Dabei wird angenommen, dass die Schwankungen der Oberflächentemperaturen jenen der Lufttemperatur entsprechen. Für Orte mit starkem Strahlungs- oder Schneeeinfluss kann dies stark vereinfacht sein. Ebenso ergänzt wurde der Einfluss der latenten Wärme beim Schmelzvorgang, der erst bei transienten Simulationen eine Rolle spielt. Dieser wurde nach MotTaghy \& RAтн (2006) mittels des Ansatzes einer scheinbaren Wärmekapazität implementiert.

\subsection{Wichtigste Inputdaten}

Das verwendetete DGM hat eine Auflösung von $20 \mathrm{~m}$ und wurde basierend auf digitalisierten Höhenlinien generiert, die im Rahmen einer photogrammetrischen Auswertung der bayerischen Landesvermessung in den 70er Jahren erhoben wurden.

Für das Energiebilanzmodell werden lückenlose Zeitreihen von Meteoparametern in stündlicher Auflösung benötigt. Lufttemperatur, Luftdruck, relative Luftfeuchtigkeit, Niederschlag, Windrichtung und -geschwindigkeit waren für die Station Zugspitze vom DWD erhältlich. Kleinere Datenlücken (im Bereich von Stunden bis Tagen) wurden mittels Interpolation zwischen den Werten vor und nach dem Messausfall gefüllt. Die Globalstrahlung wird von der Umweltforschungsstation Schneefernerhaus aufgezeichnet und ist für die Periode von 2000 bis Mitte 2006 erhältlich, was den möglichen Zeitraum der Simulation bestimmt. Die Strahlungsdaten enthalten einige grössere Lücken von bis zu mehreren Monaten. Für diese wurden jeweils alle Meteoparameter durch die Werte des Vorjahres ersetzt, damit keine unnatürlichen Klimazeitreihen generiert werden (die Werte von 2003 wurden wegen der extremen Bedingungen nicht zum Füllen von Lücken verwendet). Die Horizontabschattung der Station Schneefernerhaus ist nicht genau bekannt und wird für die Berechnung nicht korrigiert.

Für die Oberflächen- und Untergrundcharakteristika des Zugspitze-Kalksteins wurden durchschnittliche Werte aus der Literatur verwendet (z.B. CERMÁk \& Rybach 1982; Gruber et al. 2004; Wegmann et al. 1998). Die Oberflächenbedingungen wurden analog den Einstellungen für die Modellvalidierung als Albedo $=0.2$, Emissivität $=0.96$ und Oberflächenrauhigkeit $=0.0001 \mathrm{~m}$ angenommen. Die thermische Leitfähigkeit wurde auf $2.5 \mathrm{WK}^{-1} \mathrm{~m}^{-1}$ gesetzt Für die transienten Berechnungen wurden eine volumetrische Wärmekapazität von $2.0 \times 10^{6} \mathrm{Jm}^{-3} \mathrm{~K}^{-1}$ und eine Porosität von $5 \%$ angenommen.

\subsection{Resultate der Vorstudie}

Oberflächentemperaturen. Für die Oberflächentemperaturen werden an dieser Stelle die Resultate der kälteren Simulation $\mathrm{m} 2$ präsentiert, da diese den realen Verhältnissen vermutlich am besten entsprechen. Die mittleren Oberflächentemperaturen für die Periode 2000-2005 liegen zwischen $-4.5{ }^{\circ} \mathrm{C}$ in den kältesten und $6.5^{\circ} \mathrm{C}$ in den wärmsten Bereichen (Abb. 3). Die Gebiete mit negativen Temperaturen sind hauptsächlich in den nordexponierten Steilhängen zu finden, mit den tiefsten Temperaturen im oberen Bereich des Nordhangs unterhalb des Gipfels der Zugspitze. Die Temperaturdifferenz zwischen der Nord- und Südseite des Grates beträgt $4-5^{\circ} \mathrm{C}$.

Untergrundtemperaturen. Die Untergrundtemperaturen im Gipfelgrat (Abb. 4) ändern sich in erster Linie von der Nord- zur Südseite, und die Permafrostgrenze im Innern des Grates verläuft im oberen Teil nahezu senkrecht. Permafrost ist unter der Nordseite des Gipfelgrates zu erwarten, die drei Simulationen unterscheiden sich aber in seiner Mächtigkeit und Ausdehnung. Für die kälteste Situation ist auch der oberste Bereich der Südseite knapp im Permafrost gelegen (dunkelgrau und mittelgrau schattierter Bereich in Abb. 4). Auf der Nordseite sind Temperaturen bis etwa $-4{ }^{\circ} \mathrm{C}$ zu erwarten, der grösste Teil des Permafrosts hat aber eine Temperatur zwischen 0 und $-2{ }^{\circ} \mathrm{C}$. Synthetische Temperaturprofile, die beim möglichen Bohrstandort auf der Südseite aus den simulierten Temperaturfeldern extrahiert wurden, zeigen für die kälteste Simulation für das gesamte Profil Permafrost, für die 


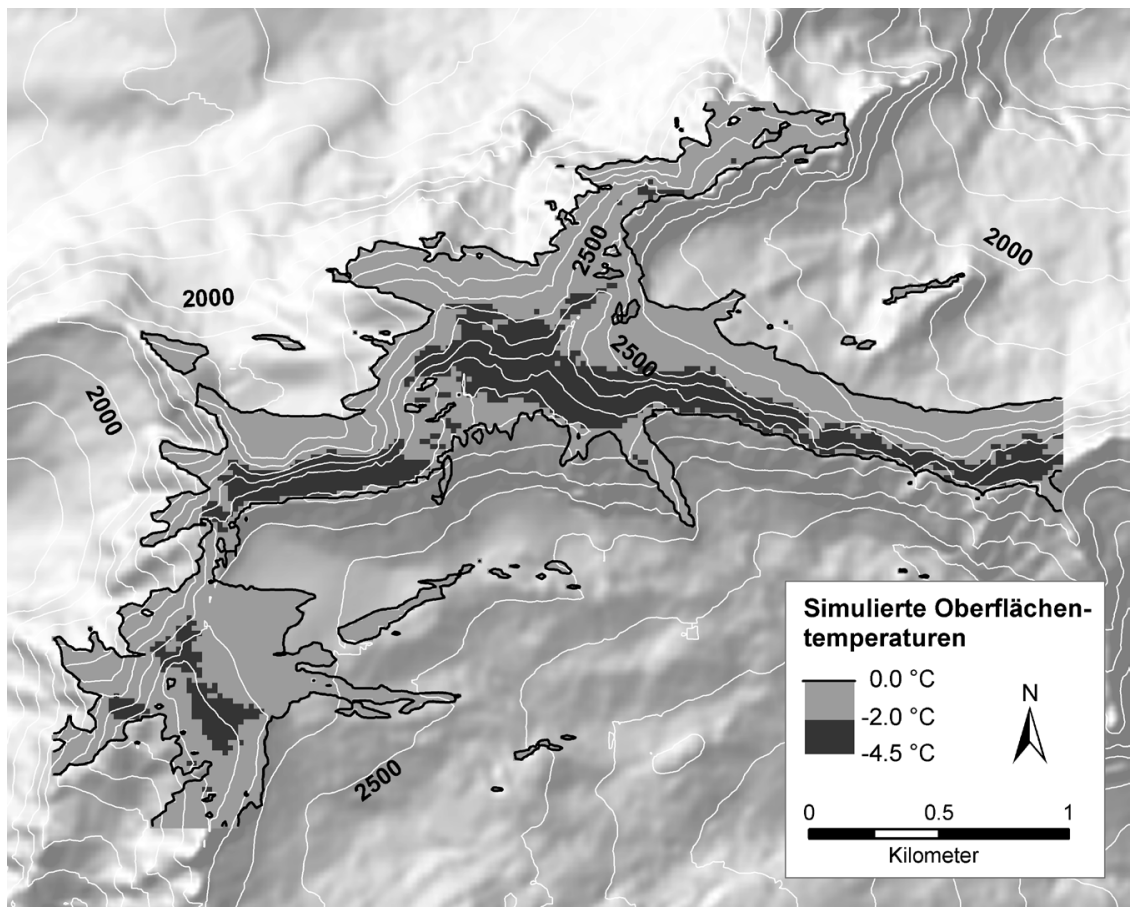

Abb. 3: Simulierte mittlere Oberflächentemperaturen 2000-2005 für m2 im Gebiet des Zugspitzgipfels Simulated mean surface temperatures 2000-2005 for the m2 scenario in the Zugspitze summit region Températures moyennes de surface simulées entre 2000 et 2005 pour la région du sommet du Zugspitze, scénario m2

mittlere Simulation erst ab ca. $40 \mathrm{~m}$ Entfernung von der Oberfläche.

Die modellierten Untergrundtemperaturen sind stark abhängig von den als Randbedingung eingesetzten Oberflächentemperaturen. Die oben beschriebenen Unsicherheiten (Abschnitt 3.1) gelten damit auch für die Untergrundtemperaturen. Dazu kommen mögliche Abweichungen zur realen Situation aufgrund der angenommenen Materialeigenschaften und Inhomogenitäten sowie der Vernachlässigung anderer Wärmetransportprozesse als der reinen Konduktion (z.B. Advektion durch zirkulierendes Wasser). Weiter wurden für die Vorstudie transiente Effekte und Phasenübergänge nicht berücksichtigt.

\subsection{Empfehlungen für Ort und Richtung der Bohrung}

Aus den oben beschriebenen Resultaten scheint es wahrscheinlich, dass mit einer horizontalen oder schrägen Bohrung von der Südseite des Gipfels Permafrost gemessen werden kann. Für die tiefsten Temperaturen sollte im obersten Bereich und so weit wie möglich in die Nordseite gebohrt werden. Im wärmsten berechneten Fall ( $\mathrm{p} 1)$ sind die tiefsten Temperaturen im Bereich von $-1{ }^{\circ} \mathrm{C}$, im kälteren Fall (m2) um $-4{ }^{\circ} \mathrm{C}$. Eine horizontale Bohrung hat den Vorteil der kürzesten Distanz, ist aber logistisch schwieriger (z.B. Auskleiden des Bohrlochs, Einführen der Thermistorenkette). Zusätzlich zur Bohrung ist eine Instrumentierung auf beiden Seiten des Grates mit Sensoren zur Messung der Oberflächentemperaturen sinnvoll für ein langfristiges Temperaturmonitoring auf dem Zugspitzgipfel (Gruber et al. 2003; HoelzLe et al. 1999).

\section{Bohrlochtemperaturen und Vergleich mit Modellresultaten}

4.1 Realisierte Bohrungen und erste Messresultate Im August 2007 wurde von der Südseite unterhalb der Gipfelstation auf einer Höhe von 2922 m ü.M. gebohrt (Abb.5). In Bohrung Ost wurde mit einem Winkel von $25^{\circ}$ zur Horizontalen bis in $58 \mathrm{~m}$ Tiefe gebohrt (vgl. Tab. 1); in Bohrung West mit $20^{\circ}$ wurde der Gipfelgrat auf einer Länge von $44 \mathrm{~m}$ ganz durchstochen. 


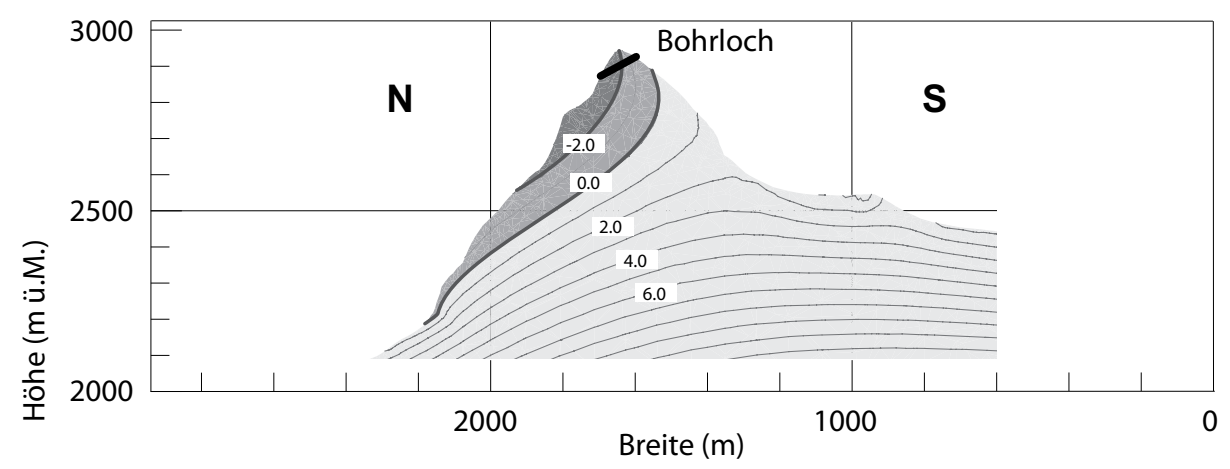

Abb. 4: Nord-Süd-Querschnitt (2D) durch das berechnete stationäre Temperaturfeld (3D) des Zugspitzgipfelgrats für die kältere Simulation $\mathrm{m} 2$

Die dunkelgrauen Linien markieren die $0{ }^{\circ} \mathrm{C}$ und die $-2{ }^{\circ} \mathrm{C}$ Isothermen. Diese entsprechen der Permafrostgrenze für das mittlere $\left(-2^{\circ} \mathrm{C}\right)$ und das kältere Szenario $\mathrm{m} 2\left(0^{\circ} \mathrm{C}\right)$. Die schattierten Bereiche zeigen den Permafrostkörper in der Zugspitze für das mittlere (dunkelgrau) und das kältere Szenario m2 (grau). Die dicke schwarze Linie zeigt die Lage des Bohrlochs West.

North-south cross section through the modeled temperature field of the summit crest of the Zugspitze for the colder simulation $m 2$

Section transversale nord-sud à travers le champ de températures modélisé pour le sommet du Zugspitze (simulation $\mathrm{m} 2$ la plus froide)

Eine Thermistorenkette mit 25 Sensoren (Genauigkeit $\pm 0.1{ }^{\circ} \mathrm{C}$ )wurde im Bohrloch West installiert, und deren Messungen werden stündlich automatisch aufgezeichnet. Die Messdaten der ersten zwei Jahre sind in Abb. 6 und 7 visualisiert. Im Bereich des Bohrlochs ist der Gipfelgrat komplett im Permafrost gelegen. Jahreszeitliche Schwankungen dringen von Norden und Süden her in den Untergrund und sind bis in eine Tiefe von jeweils ca. $15 \mathrm{~m}$ bemerkbar. Die Felstempe- raturen, die nicht von jahreszeitlichen Schwankungen beeinflusst werden, liegen im Bereich von -4 bis $-3^{\circ} \mathrm{C}$. Die tiefsten Temperaturen werden an der Oberfläche im Norden (um $-12{ }^{\circ} \mathrm{C}$ ), die höchsten entsprechend im Süden (um $+15^{\circ} \mathrm{C}$ ) gemessen. Da das Bohrloch den Grat vollständig durchsticht, wird auf zwei Seiten eine Auftauschicht gemessen. Auf der Südseite ist die jährliche Auftauschicht ca. $4.5 \mathrm{~m}$, auf der Nordseite um $1.5 \mathrm{~m}$ (vgl.Tab. 2). Im Temperaturprofil fällt der Sprung
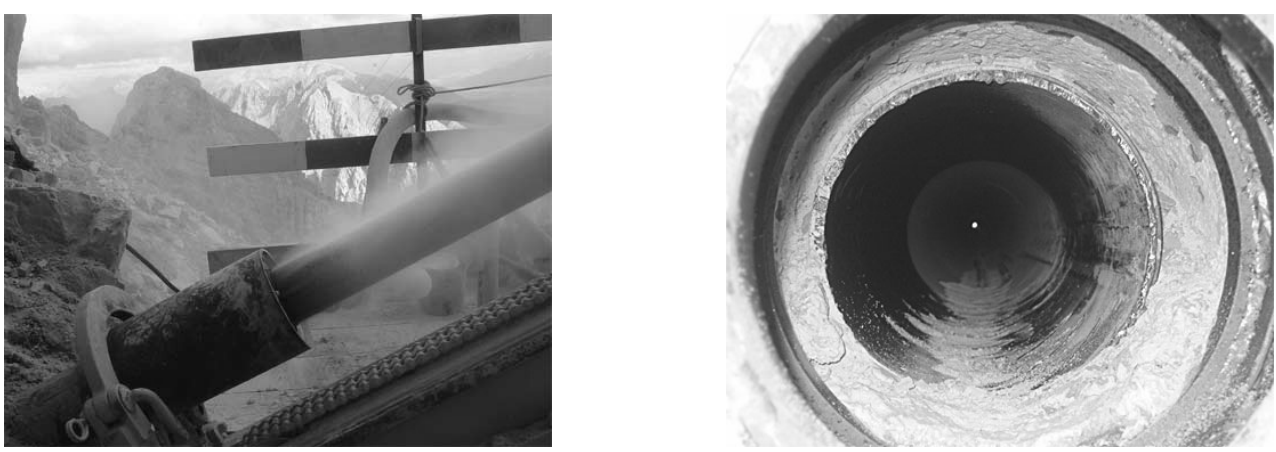

Abb. 5. Bohrarbeiten auf der Zugspitze im August 2007 (links) und das Bohrloch West (rechts) Drilling work on the Zugspitze in August 2007 (left) and the completed Borehole West (right) Travaux de forage au Zugspitze en août 2007 (à gauche) et forage ouest terminé (à droite) Fotos: www.stump.ch (Zugriff im März 2008) 


\begin{tabular}{|l|l|l|l|l|}
\hline & Winkel/Länge & Koordinaten & \#Thermistoren & Weiteres \\
\hline Bohrloch West & $20^{\circ} / 44 \mathrm{~m}$ & RW: 4423582 & 25 & $\begin{array}{l}\text { Durchsticht den } \\
\text { Grat komplett } \\
\text { ZUG_0107 }\end{array}$ \\
Gebohrt: 8.2007 & & HW: 5254102 & & $\begin{array}{l}\text { Weitere } \\
\text { Instrumente: } \\
\text { Extensometer }\end{array}$ \\
\hline Bohrloch Ost & $25^{\circ} / 58 \mathrm{~m}$ & RW: 4423583 & 3 & Durchsticht den \\
ZUG_0207 & & HW: 5254102 & & $\begin{array}{l}\text { Grat nicht } \\
\text { Instrumente: } \\
\text { Gebohrt: } 8.2007\end{array}$ \\
& & Z: 2922 & & Fiber-Optik-Kabel \\
\hline
\end{tabular}

Tab. 1: Beschreibung der Bohrlöcher im Gipfelgrat der Zugspitze. \#Therm. ist die Anzahl Thermistoren. Description of the boreholes in the summit crest of the Zugspitze mountain. \#Therm. is the number of thermistors. Description des forages réalisés sur l'arête sommitale du Zuspitze. \#Therm. indique le nombre de thermistors.

und anschliessende Zickzack in den Mitteltemperaturen der obersten Sensoren (Südseite) auf. Der Grund können Messfehler oder eine tatsächliche thermische Störung sein. Eine Erklärung wäre eindringendes
Schmelzwasser, das im Winter die Temperaturen bei $0{ }^{\circ} \mathrm{C}$ hält und $\mathrm{zu}$ hohen Mitteltemperaturen führt. In diesem Fall müsste die Verrohrung, welche die obersten $5 \mathrm{~m}$ abdeckt, überprüft werden.

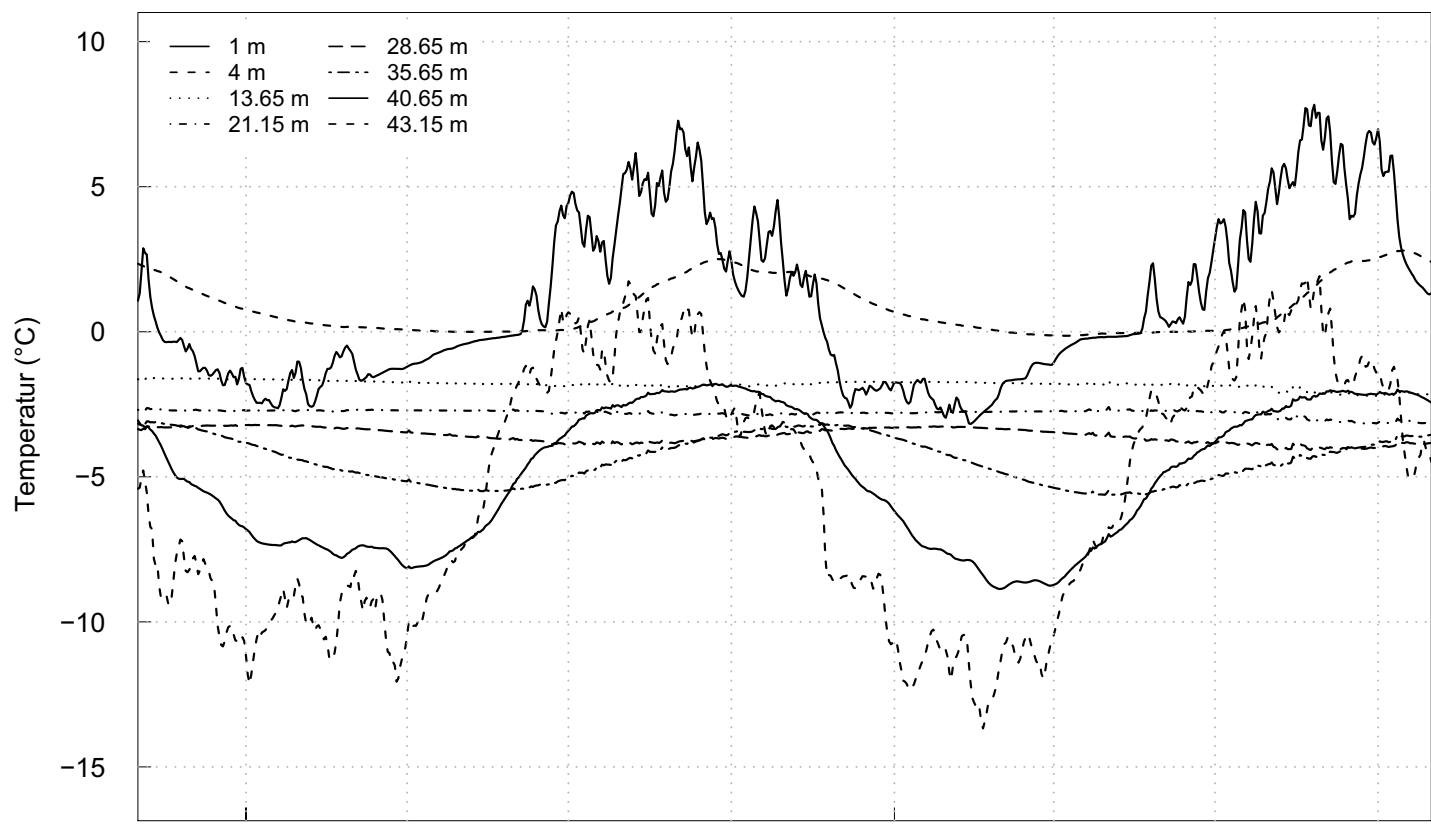

Jan. 08

Jan. 09

Abb. 6: Temperatur-Zeit-Plot für das Bohrloch West für die Periode Nov. 2007 bis Nov. 2009. Die Tiefe von 0 m entspricht der Oberfläche auf der Südseite, jene von 44 m der Oberfläche auf der Nordseite.

Temperature time plot for Borehole West for the time period Nov. 2007 to Nov. 2009. The surface on the south side is at $0 \mathrm{~m}$, the depth of $44 \mathrm{~m}$ corresponds to the surface on the north side.

Courbe des températures du forage ouest pour la période allant de novembre 2007 à novembre 2009. La surface sur le côté sud est à $0 \mathrm{~m}$ et la profondeur de $44 \mathrm{~m}$ correspond à la surface du côté nord. 


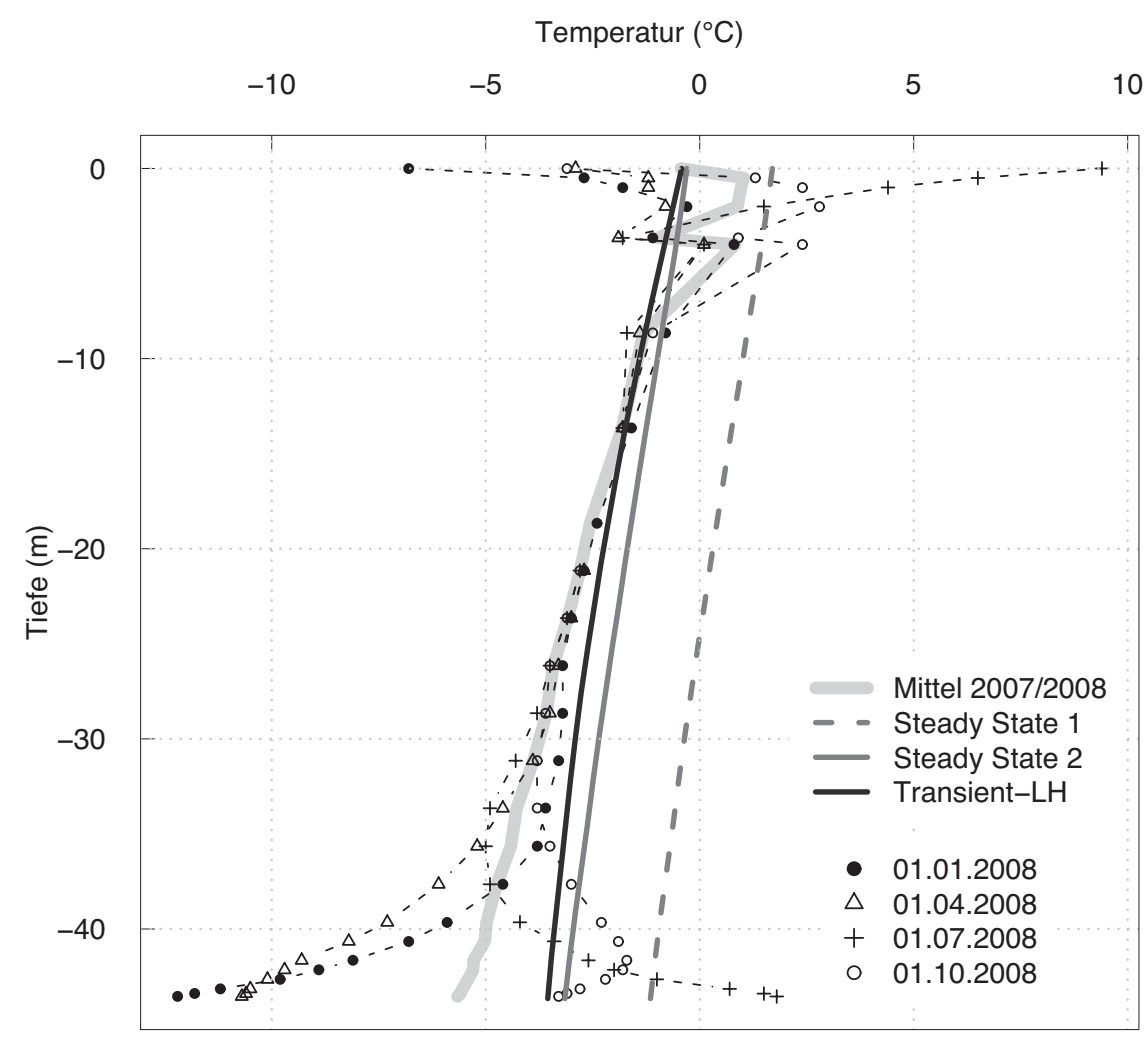

Abb. 7. Gemessene Profile aus dem Bohrloch West (gestrichelt mit Punkten), verglichen mit modellierten Profilen (dunkelgraue Linien)

Für die Bohrlochdaten ist der Mittelwert 2007-2009 in hellgrau eingezeichnet. «Steady State 1» ist das simulierte stationäre Temperaturprofil, «Steady State 2» jenes für die kälteren Bedingungen m2, «Transient-LH» das transiente Temperaturprofil mit Berücksichtigung von latenter Wärme.

Measured profiles in the Borehole West (dashed lines with points) compared with modeled profiles (dark grey lines) Profils mesurés dans le forage ouest (tirets avec points) comparés avec les profils modélisés (lignes en gris foncé)

\subsection{Vergleich der Bohrlochdaten mit den simulierten Temperaturen}

In Abb. 7 sind die Temperaturprofile im Bohrloch West für vier Zeitpunkte im Jahr 2008 zusammen mit den simulierten Profilen dargestellt. Wie erwartet, ist das Resultat der Vorstudie, das auf Meteodaten von 20002005 basiert, zu warm. Das Profil des um $-2{ }^{\circ} \mathrm{C}$ korrigierten Resultats (m2) stimmt mit den Messdaten besser überein und ist im mittleren Bereich des Bohrlochs etwa $1{ }^{\circ} \mathrm{C}$, im nördlichen Teil etwa $2^{\circ} \mathrm{C}$ wärmer als gemessen. Der Temperaturgradient von der Nord- zur Südseite, und damit der Unterschied der Oberflächentemperaturen, wurde in der Simulation leicht unterschätzt.

Um den Fehler abzuschätzen, der aus der Vernachlässigung zeitabhängiger Effekte und latenter Wärme resultiert, wurde zusätzlich eine transiente Simulation gemacht. Das resultierende Temperaturprofil zeigt ebenfalls leicht höhere Temperaturen im Vergleich zum Bohrloch. Die Unterschiede sind aber mehrheitlich unter $1{ }^{\circ} \mathrm{C}$ und in dem nicht von saisonalen Schwankungen beeinflussten Teil (zwischen etwa 12 und $32 \mathrm{~m}$ ) im Bereich von $0.5^{\circ} \mathrm{C}$.

\section{Schlussfolgerungen}

Im Rahmen einer Vorstudie für die Bohrung auf der Zugspitze wurden die Temperatur- und Permafrostverhältnisse im Gipfelgrat mit Modellen untersucht. Im Sommer 2007 wurden zwei Bohrungen von der Südseite des Grates gemacht, von denen eine den Grat auf 


\begin{tabular}{|l|l|l|l|l|}
\hline Jahr & Max. ALT Süd & Datum & Max. ALT Nord & Datum \\
\hline 2008 & $4.38 \mathrm{~m}$ & 26.09 .2008 & $1.51 \mathrm{~m}$ & 15.08 .2008 \\
2009 & $\ldots$ & 18.10 .2008 & $1.76 \mathrm{~m}$ & 30.08 .2009 \\
\hline
\end{tabular}

Tab. 2: Tiefe der Auftauschicht (ALT) auf der Süd- und Nordseite des Bohrlochs West und entsprechendes Datum

Active layer thickness (ALT) on the south and north side of Borehole West and corresponding date

Epaisseur de la couche active (ALT) pour les faces nord et sud du forage ouest (avec la date correspondante)

einer Länge von $44 \mathrm{~m}$ schräg durchstösst. Die Resultate der Simulationen, die erste Analyse der Messdaten und der Vergleich der beiden ergeben folgendes Bild:

- Der Gipfelgrat der Zugspitze liegt im Grenzbereich des Permafrostvorkommens. Die Permafrostgrenze verläuft südlich nahe dem Grat und im Inneren des Grates beinahe senkrecht.

- Die Oberflächentemperaturen unterscheiden sich zwischen Nord- und Südseite um 4-5 ${ }^{\circ} \mathrm{C}$. Im Inneren des Grates werden Permafrosttemperaturen im Bereich von 0 bis $-4{ }^{\circ} \mathrm{C}$ gemessen.

- Die simulierten Temperaturen der Vorstudie liegen für den kälteren angenommenen Fall im Bereich der gemessenen Werte, sind generell aber $1-2{ }^{\circ} \mathrm{C}$ zu hoch. Da die nördliche Randbedingung relativ zur südlichen zu warm berechnet wurde, wurde der Temperaturgradient leicht unterschätzt. Die Temperaturverteilung im Grat wurde aber generell gut wiedergegeben.

- Die Ergänzung der Simulation durch transiente Effekte und die Berücksichtigung von latenter Wärme hat das Modellresultat verbessert.

Mittels einer 3D-Temperatur-Modellierung konnten die Permafrostverhältnisse im Felsgrat der Zugspitze im Vorfeld einer Bohrung zufriedenstellend charakterisiert werden und Hinweise darauf geben, wo und in welche Richtung gebohrt werden sollte. Voraussetzung dafür waren das Vorhandensein der Inputdaten (DHM und Meteozeitreihen) sowie die relativ einfach zu modellierenden thermischen Verhältnisse in steilem Fels.

\section{Ausblick}

Das Permafrost-Monitoring auf der Zugspitze wird vom LfU weitergeführt und im Rahmen des EU-Interreg-Projekts «PermaNET» weiter ausgebaut.Das Bohrloch auf der Zugspitze ergänzt das Permafrost-Monitoring im Alpenraum um einen wichtigen Standort in den Nordalpen. Im Umfeld des Bohrlochs wurden im Sommer 2008 und 2009 auf beiden Seiten des Grates Sensoren zur Messung der Oberflächentemperaturen installiert. Solche Messungen sind aus mehreren Grün- den wichtig: die Thermistoren nahe der Oberfläche können durch die Bohrung oder das Gebäude gestört sein, die Temperaturen im Untergrund können je nach Tiefe von einer grösseren Oberfläche beeinflusst werden, ferner ist der oberste Thermistor allein weniger repräsentativ, insbesondere auch im Hinblick auf den Einfluss von Schnee, der auf der Südseite liegen bleibt. Solche Messungen erlauben auch, die räumliche Variabilität im Gebiet abzuschätzen und Veränderungen im Untergrund zu antizipieren. Im Rahmen dieses Projekts werden die Modellierungen erweitert und Simulationen angestrebt, die den Einfluss einer Schneedecke und den Einfluss von Phasenübergängen in gesättigtem und ungesättigtem Lockermaterial sowie im zerklüfteten Fels abschätzen. Diese Prozesse sind vermutlich besonders auf der Südseite wichtig. In Gipfelnähe wurde zudem durch die Universität Bonn ein Geoelektrik-Monitoring eingerichtet, das Auskunft gibt über Veränderungen von Eis und ungefrorenem Wassergehalt im Fels und somit ergänzende Informationen zum Temperatur-Monitoring liefert (HiLbich et al. 2008; Krautblatter \& Hauck 2007).

\section{Dank}

Die Permafrost-Messungen auf der Zugspitze werden durch das EU-Programm «Territorial Cooperation Alpine Space 2007-2013» im Projekt PermaNET unterstützt.

\section{Literatur}

Cermák, V. \& L. Rybach (1982): Thermal conductivity and specific heat of minerals and rocks. - In: LandoltBörnstein Zahlenwerte und Funktionen aus Naturwissenschaften und Technik, Neue Serie, Physikalische Eigenschaften der Gesteine (V/1a). - Berlin: Springer: 305-343.

Fischer, L., KäÄв, A., Huggel, C. \& J. NöTZli (2006): Geology, glacier retreat and permafrost degradation as controlling factors of slope instabilities in a high-mountain rock wall: the Monte Rosa east face. - In: Natural Hazards and Earth System Sciences 6: 761-772. 
FISCHER, L. (2009): Slope instabilities on perennially frozen and glacierised rock walls: multiscale observation, analyses and modelling. - Dissertation, University of Zurich, Department of Geography.

Gruber, S. (2005): Mountain permafrost: Transient spatial modelling, model verfication and the use of remote sensing. - Dissertation, University of Zurich, Department of Geography.

Gruber, S. \& W. Haeberli (2007): Permafrost in steep bedrock slopes and its temperature-related destabilization following climate change. - In: Journal of Geophysical Research 112: doi:10.1029/2006JF000547.

Gruber, S., Peter, M., Hoelzle, M., Woodhatch, I. \& W. Haeberli (2003): Surface temperatures in steep alpine rock faces - A strategy for regional-scale measurement and modelling. - In: Proceedings of the $8^{\text {th }}$ International Conference on Permafrost, 21-25 July 2003, Zürich: 325-330.

Gruber, S., Hoelzle, M. \& W. Haeberli (2004): Rockwall temperatures in the Alps: modelling their topographic distribution and regional differences. - In: Permafrost and Periglacial Processes 15, 3: 299-307.

Gude, M. \& D. BARSCH (2005): Assessment of geomorphic hazards in connection with permafrost occurrence in the Zugspitze area (Bavarian Alps, Germany). - In: Geomorphology 66, 1-4: 85-93.

Haeberli, W., Wegmann, M. \& D. Vonder Mühll (1997): Slope stability problems related to glacier shrinkage and permafrost degradation in the Alps. - In: Eclogae Geologicae Helvetiae 90: 407-414.

Hasler, A., Talzi, I., Beutel, J., Tschudin, C. \& S. GrUber (2008): Wireless sensor networks in permafrost research - concept, requirements, implementation and challenges. - In: Proceedings of the $9^{\text {th }}$ International Conference on Permafrost, June 29-July 3, 2008, Fairbanks: 669-674.

Hilbich, C., Hauck, C., Hoelzle, M., Scherler, M., Schudel, L., VölKsch, I., Vonder MÜHLl, D. \& R. MäUSBACHER (2008): Monitoring mountain permafrost evolution using electrical resistivity tomography: A 7-year study of seasonal, annual, and long-term variations at Schilthorn, Swiss Alps. - In: Journal of Geophysical Research 113: doi:10.1029/2007JF000799.

Hoelzle, M., Wegmann, M. \& B. Krummenacher (1999): Miniature temperature dataloggers for mapping and monitoring of permafrost in high mountain areas: first experience from the Swiss Alps. - In: Permafrost and Periglacial Processes 10, 2:113-124.

Keller, F. (2003): Kurzbericht über die Steinschlagereignisse im heissen Sommer 2003 im Bergell (Project report on rock fall 2003 to the canton Graubünden). - Samedan: Institut für Tourismus und Landschaft, Academia Engiadina.

KöRNER, H. \& R. ULRICH (1965): Geologische und felsmechanische Untersuchungen für die Gipfelstation der Seilbahn Eibsee-Zugspitze. - In: Geologica Bavarica 55: 404-421.
Krautblatter, M. \& C. Hauck (2007): Electrical resistivity tomography monitoring of permafrost in solid rock walls. - In: Journal of Geophysical Research 112: doi:10.1029/2006JF000546.

Medici, F. \& L. Ryвасн (1995): Geothermal map of Switzerland 1995 (heat flow density). - In: Beiträge zur Geologie der Schweiz. Geophysik 30: 36.

MotTaghy, D. \& V. Rath (2006): Latent heat effects in subsurface heat transport modeling and their impact on paleotemperature reconstructions. - In: Geophysical Journal International 164: 236-245.

Mustafa, O. (2001): Persönliche Kommunikation. - Institut für Geographie, Universität Jena.

Mustafa, O., Gude, M. \& M. Hoelzle (2003): Modeling permafrost distribution in the northern Alps using global radiation. $-8^{\text {th }}$ International Conference on Permafrost, Extended Abstracts, Zürich, Switzerland, 111-112.

NöTZLI, J.(2008): Modeling transient three-dimensional temperature fields in mountain permafrost. - Dissertation, University of Zurich, Department of Geography. NöTZLI, J. \& S. GrUber (2009): Transient thermal effects in Alpine permafrost. - In: The Cryosphere 3: 85-99.

Nötzli,J.,Gruber, S. \& W.HAeberli (2006):3D-Modellierung der thermischen Bedingungen im Bereich des Gipfelgrates der Zugspitze. - Zürich: Geographisches Institut, Universität Zürich.

Nötzli, J., Gruber, S., Kohl, T., Salzmann, N. \& W. HAEBERLI (2007): Three-dimensional distribution and evolution of permafrost temperatures in idealized high-mountain topography. - In: Journal of Geophysical Research 112: doi:10.1029/ 2006JF000545.

Nötzli, J., Hilbich, C., Hauck, C., Hoelzle, M. \& S. GrUBER (2008): Comparison of simulated 2D temperature profiles with time-lapse electrical resistivity data at the Schilthorn crest, Switzerland. - In: Proceedings of the $9^{\text {th }}$ International Conference on Permafrost, June 29-July 3, 2008, Fairbanks: 1293-1298.

Nötzli, J., Hoelzle, M. \& W. Haeberli (2003): Mountain permafrost and recent Alpine rock-fall events: a GIS-based approach to determine critical factors. - In: Proceedings of the $8^{\text {th }}$ International Conference on Permafrost, 21-25 July 2003, Zürich: 827-832.

Ravanel, L. (2006): Contribution à l'étude des écroulements dans les parois à permafrost de la haute montagne alpine - L'example du Petit Dru (massif du Mont Blanc) depuis la fin du Petit Age Glaciaire. - Unveröffentlichte Diplomarbeit, Laboratoire EDYTEMUMR 5204, Université de Savoie, Chambéry.

Sosio, R., Crosta, G. \& O. Hungr (2008): Complete dynamic modeling calibration for the Thurwieser rock avalanche (Italian Central Alps). - In: Engineering Geology: doi: 10.1016/j.enggeo.2008.02.012.

UlRICH, R. \& L. KING (1993): Influence of mountain permafrost on construction in the Zugspitze mountains, Bavarian Alps, Germany. - In: Proceedings of the $6^{\text {th }}$ International Conference on Permafrost, July 5-9, 1993, Beijing: 25-630. 
Wegmann, M., Gudmundsson, G.H. \& W. Haeberli (1998): Permafrost changes in rock walls and the retreat of alpine glaciers: a thermal modelling approach. - In: Permafrost and Periglacial Processes 9, 1:23-33.

\begin{abstract}
Zusammenfassung: Modellierung und Messung von Permafrosttemperaturen im Gipfelgrat der Zugspitze, Deutschland

Im Gebiet der Zugspitze, dem mit 2962 m ü.M. höchsten Berg Deutschlands, sind auch die bedeutendsten Permafrostvorkommen des Landes zu finden. Der Felsgrat der Zugspitze ist steil und stark bebaut. Schon während der Bauarbeiten in den 1960er Jahren wurden massives Eis in Klüften und gefrorene Lockersedimente entdeckt. Allfällige Stabilitätsprobleme durch Permafrostdegradation können entscheidende Konsequenzen auf Bauwerke haben. Deshalb wurde im August 2007 für langfristige Permafrostbeobachtung eine Bohrung quer durch den Gipfelgrat gemacht und mit einer Thermistorenkette ausgestattet. In einer Vorstudie wurden die Temperaturverhältnisse mit Computermodellen simuliert. Resultate zeigen Permafrost mit Temperaturen bis etwa $-3{ }^{\circ} \mathrm{C}$ in der Nordseite und in Teilen der Südseite. Die Permafrostgrenze verläuft im Inneren des Grates und ist im oberen Bereich fast senkrecht. Die nach der Realisierung der Bohrung gemessenen Temperaturen sind etwas niedriger als im Modell, stimmen aber im Allgemeinen gut mit den Ergebnissen der Simulation überein, insbesondere wenn auch transiente Effekte und der Einfluss latenter Wärme berücksichtigt werden. Monitoring und Modellstudien des Permafrosts in der Zugspitze werden im Rahmen von internationalen Projekten weitergeführt und ausgebaut.
\end{abstract}

Schlüsselwörter: Felspermafrost, dreidimensionale Modellierung, Bohrlochtemperaturen, Zugspitze

\section{Summary: Modeling and measurement of permafrost temperatures in the summit crest of the Zugspitze, Germany}

The most important permafrost occurrences in Germany are found in the region of the Zugspitze $(2962 \mathrm{~m}$ a.s.l.), Germany's highest mountain. The summit crest is very steep and considerable infrastructure is built on the top. During construction massive ice and frozen sediments were found. Possible stability problems as a result of permafrost degradation can have severe consequences. In August 2007, a borehole was drilled through the crest for long-term monitoring of permafrost conditions. The temperature conditions in the crest were modeled in a preparatory study. Results show permafrost with temperatures down to $-3{ }^{\circ} \mathrm{C}$ on the northern side and in parts of the southern side. The permafrost boundary is within the crest and nearly vertical in the upper part. Temperatures measured in the borehole show somewhat colder temperatures, but generally agree well with the model results, especially when the simulation includes transient effects and latent heat. Monitoring and modeling studies of the permafrost on the Zugspitze are being continued and extended in the scope of international projects.

Keywords: bedrock permafrost, three-dimensional modeling, borehole temperatures, Zugspitze

\section{Résumé: Modélisation et mesures des températures} du pergélisol au sommet du Zugspitze (Allemagne) La présence la plus importante de pergélisol en Allemagne se trouve dans la région du Zugspitze (2962 m, point culminant du pays). On trouve à cet endroit de grandes infrastructures construites sur une crête très abrupte. Durant les travaux de construction, des masses de glace ainsi que des sédiments gelés ont été déblayés. Une dégradation du pergélisol à cet endroit, entraînant de possibles problèmes d'instabilité, aurait des conséquences sérieuses. En août 2007, un forage à travers la crête a été entrepris pour permettre une étude à long terme des conditions du pergélisol. Le pergélisol a été modélisé dans une étude préparatoire. Les résultats montrent que le pergélisol est présent avec des températures allant de $-3{ }^{\circ} \mathrm{C}$ à $0{ }^{\circ} \mathrm{C}$ sur la face nord et quasiment absent sur la face sud. La limite du pergélisol se trouve sous la crête et est quasiment verticale dans la partie supérieure. Les températures mesurées dans le forage montrent, d'une certaine façon, des valeurs de température plus faibles mais généralement en accord avec les résultats des modélisations, particulièrement lorsque les simulations incluent les effets transitoires et la chaleur latente. La surveillance et la modélisation du pergélisol au Zugspitze continuent et sont développées dans le cadre de projets internationaux.

Mots-clés: pergélisol en milieu rocheux, modélisation 3D, températures de forage, Zugspitze

Dr. Jeannette Nötzli, Dr. Stephan Gruber, Geographisches Institut, Universität Zürich, Winterthurerstrasse 190, CH-8057 Zürich, Schweiz.

e-mail:

jeannette.noetzli@geo.uzh.ch

stephan.gruber@geo.uzh.ch

Dr. Andreas von Poschinger, Bayerisches Landesamt für Umwelt, Lazarettstrasse 67, D-80636 München, Deutschland.

e-mail: andreas.poschinger@lfu.bayern.de

\section{Manuskripteingang/received/manuscrit entré le} 25.1.2010

Annahme zum Druck/accepted for publication/accepté pour l'impression: 22.7.2010 